\title{
Churg-Strauss syndrome presenting with focal segmental glomerulosclerosis without proteinuria: response to unconventional therapy
}

\author{
Pradipta Guha ${ }^{1}$, MD, Indranil Thakur $^{2}$, MBBs, Arindam Ray ${ }^{2}$, mBBs, Sanjoy Kumar Chatterjee ${ }^{2}$, MD
}

\begin{abstract}
Churg-Strauss syndrome (CSS), or allergic granulomatosis, is a rare disease manifested by tissue infiltration, hypereosinophilia and vasculitis. Renal involvement may be seen in up to $50 \%$ of cases. We report the case of a 25 -yearold man who presented with a history of fever for two months, tingling, numbness, and paraesthesia of the upper limbs and left lower limb, along with diarrhoea for one month and an inability to walk for the past seven days. Serial laboratory investigations helped to reach the final diagnosis of CSS with mononeuritis multiplex, and skin, pulmonary and gastrointestinal involvement with hypertension. This is due to renal involvement in the form of focal segmental glomerulosclerosis without any nephrotic range proteinuria, which is a very rare clinical entity. The patient's symptoms were relieved after the administration of an unconventional mode of therapy.
\end{abstract}

Keywords: Churg-Strauss syndrome, focal segmental glomerulosclerosis, immunosuppressive therapy

Singapore Med J 2013; 54(1): e13-e15

\section{INTRODUCTION}

Churg-Strauss syndrome (CSS) should be considered when there is late onset of asthma, worsening asthma or a rash, along with either numbness or pain in the extremities, a lingering cough, sinus problems, stomach problems, or symptoms suggestive of cardiac involvement. In an autopsy series, the kidney is affected in over $50 \%$ of cases and clinical renal involvement has been described in $25 \%$ to more than $90 \%$ of cases. ${ }^{(1)}$ In a review study, only 21 cases of CSS were identified among almost 185,000 asthmatic

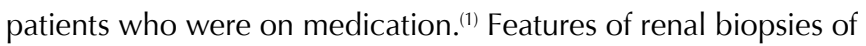
CSS vary widely from normal kidney tissue to vasculitis, severe glomerulonephritis and interstitial inflammation. ${ }^{(2)}$ Crescentic glomerulonephritis and focal necrotising glomerulonephritis are the most commonly encountered renal lesions in CSS, affecting men slightly more than women. It can affect people of all ages, with the average age at diagnosis ranging from 35-45 years. Human eosinophil cationic proteins, which are capable of tissue destruction in a variety of hypereosinophilic syndromes, have been found in granulomatous tissue in patients with CSS. ${ }^{(3,4)}$ Although therapeutic trials in CSS are limited, they have shown that glucocorticoid alone and glucocorticoid combined with cyclophosphamide are both effective. ${ }^{(5)}$

\section{CASE REPORTS}

A 25-year-old man presented with a history of fever for the past two months. The patient complained of tingling, numbness and paraesthesia of both the upper limbs and the left lower limb over a period of one month, and an inability to walk for the past seven days. This was associated with persistent diarrhoea for the past one month. He was diagnosed with bronchial asthma and

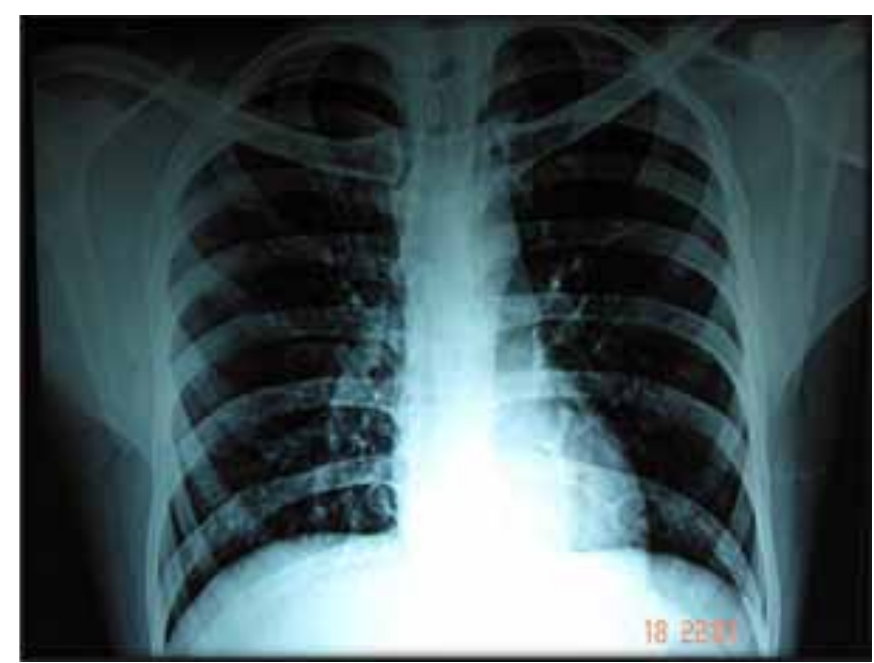

Fig. 1 Chest radiograph shows diffuse, multiple, nonspecific nodular infiltrates.

allergic rhinitis two years ago. His peripheral blood smear then had shown a high eosinophil count and spirometry had been consistent with moderate obstruction with significant reactivity. He was discharged with a prescription of an inhaler and a short course of oral steroids. After 18 months of being symptom-free, he again developed a minimally productive cough with an evening rise of low-grade temperature without chills or rigor. Sputum for an acid fast bacilli smear report was negative and the chest radiograph revealed diffuse, multiple, nonspecific nodular infiltrates (Fig. 1). His consulting physician had started him on empirical antitubercular drugs (ATD), keeping in mind the provisional diagnosis of pulmonary tuberculosis, as it is highly prevalent in this geographical region. After the administration of ATD for one month, the patient developed tingling, numbness and paraesthesia

${ }^{1}$ Department of General Medicine, Calcutta National Medical College and Hospital, ${ }^{2}$ Department of General Medicine, Medical College, Kolkata, India Correspondence: Dr Pradipta Guha, Assistant Professor, Department of General Medicine, Calcutta National Medical College and Hospital, 335, Nandan Nagar, Belgharia, Kolkata 700083, West Bengal, India. dr.pradipta@yahoo.co.in 
in the right upper limb, followed by the left upper limb, and then the lower limb. Subsequently, he developed severe weakness in the distal part of the upper limbs and left lower limb, resulting in an inability to move the limbs and a difficulty in walking. His skin also started to discolour.

At the patient's current admission, hypopigmented, oval to circular, nonpruritic and slightly elevated skin lesions of various diameters appeared on his left leg and foot. One month before this admission, he had developed large-volume, watery diarrhoea 6-7 times per day, which was not associated with tenesmus, pain in the abdomen or passage of blood with stools. There was no history of headache, dysuria, nausea, vomiting, abdominal pain, night sweats, or haemoptysis. He was a nonsmoker and nonalcoholic. He was not known to be diabetic or hypertensive. He had no past history of any major surgery, medical illness, joint pain, oro-genital ulcer or contact with tuberculosis. There was also no history of intake of aspirin, nonsteroidal anti-inflammatory drugs (NSAID) or other drugs. He had a persistently high eosinophil count throughout the past two years.

On admission, his pulse rate was 120 beats per minute with a regular pulse beat, and his blood pressure was 140/90 $\mathrm{mmHg}$. Chest auscultation revealed bilateral rhonchi with a loss of vibration sense over the left great toe. Blood report at that time showed haemoglobin level at $153 \mathrm{~g} / \mathrm{L}$, total leukocyte count at $32.2 \times 10^{9}$ cells $/ \mathrm{L}$, neutrophils at $42 \%$, lymphocytes at $19 \%$, monocytes at $4 \%$, eosinophils at $35 \%$, erythrocyte sedimentation rate (ESR) at $36 \mathrm{~mm} / \mathrm{hr}$ in the first hour, mean corpuscular volume at $79 \times 10^{-15} \mathrm{~L}$, with normal serum glucose level, liver function and renal function tests. Routine urine examination showed no proteinuria and few red blood cells. 24-hour urine protein was at $0.25 \mathrm{~g}$ /day. Serum lactate dehydrogenase was at 1,460 U/L, but serum uric acid level was normal. Stool examination revealed no ova, parasites or cysts. Serum immunoglobulin E (IgE) was at $2.2 \mathrm{mg} / \mathrm{L}$ (normal range $<0.4 \mathrm{mg} / \mathrm{L}$ ). Allergen skin test was negative for all fungal infections. Rheumatoid factor and antistreptolysin $\mathrm{O}$ titre were also negative. Ultrasonography of the whole abdomen did not reveal any abnormality. Pulmonary function test revealed moderate obstruction with significant reactivity and reversible obstructive pattern. A repeat chest radiograph showed normal study - previously detected multiple nodular opacities were now absent. The patient was HIV negative. Serum perinuclear antineutrophil cytoplasmic ( $p$-ANCA) bodies was positive (1:10 titre by immunofluorescence [IF]). The patient then developed right wrist drop. Central nervous system examination revealed weakness of the left ankle dorsiflexors, with foot drop and loss of ankle jerk. All modalities of sensory impairment of the lateral aspect of the leg extending to the dorsum of the foot, with early wasting of the small muscles of the hands, were also noted. No thickened peripheral nerve was found. With a suspicion of mononeuritis multiplex, we performed a nerve conduction velocity (NCV) test and a sural nerve biopsy. The patient's NCV test result was consistent with axonal sensorimotor polyneuropathy and his kidney biopsy

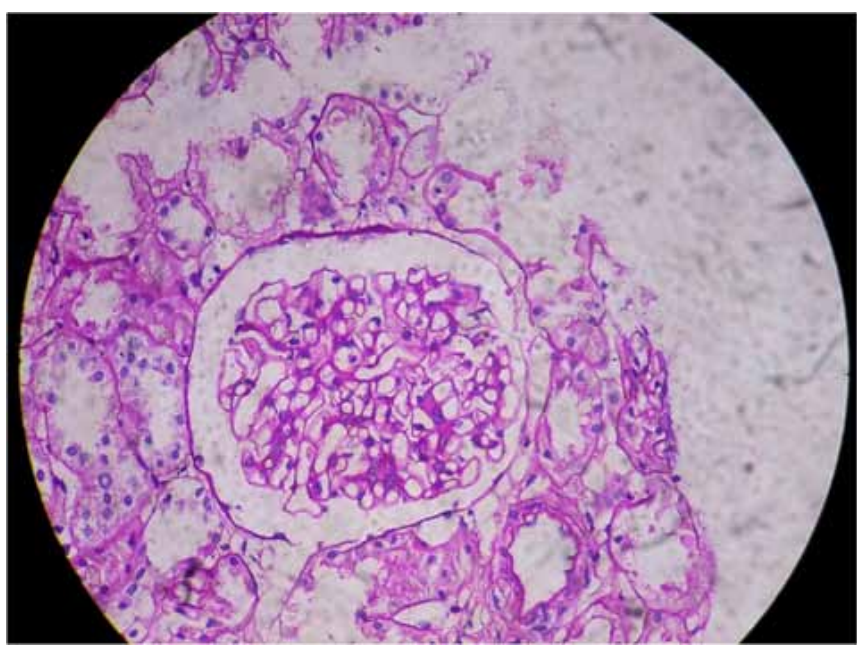

Fig. 2 Photomicrograph of kidney biopsy report shows focal segmental glomerulosclerosis without any crescents (Periodic acid-Schiff, × 200).

report revealed focal segmental glomerulosclerosis without any crescents (Fig. 2). The IF study revealed immunoglobulin M (IgM) and Complement 3 positivity in a granular, mesangial, global and diffuse pattern. Sural nerve biopsy revealed slight vasculitic changes although no extravascular granuloma was detected. During this period, the patient's blood pressure was gradually increasing (150/90 $\mathrm{mmHg}$ ). Therefore, we started him on oral enalapril at $5 \mathrm{mg} /$ day. Echocardiograhy was consistent with concentric left ventricular hypertrophy. In a setting of persistent eosinophilia with bronchial asthma-like presentation, fleeting pulmonary opacities and mononeuritis multiplex positive for p-ANCA, we finally diagnosed the case as Churg-Strauss syndrome with moderate hypertension based on the criteria by the American College of Rheumatology (ACR).

The patient was put on oral cyclophosphamide and intravenous methylprednisolone, followed by oral prednisolone. Although his diarrhoea was controlled within a few days of conservative therapy, his eosinophil count remained persistently high and no improvement of disabilities was clinically documented even after taking adequate measures for almost three weeks. When oral cyclophosphamide was switched to intravenous cyclophosphamide, his eosinophil count dropped dramatically within a few days, with a decrease in ESR. The patient has since completed five doses of pulse cyclophosphamide and is now doing well with improvements in weakness and sensory symptoms.

\section{DISCUSSION}

Churg and Strauss first described CSS in 1951. In their original article, they described three pathological features of the disease - eosinophilic tissue infiltration, granuloma formation and necrotising vasculitis that primarily involves small- and mediumsized vessels. ${ }^{(6)}$ Asthma, one of the important features of CSS, may appear a long time before the clinical appearance of vasculitis. Our patient had developed gastrointestinal vasculitis, probably in the form of diarrhoea, and mononeuritis multiplex in the form of tingling, numbness, wrist drop and wasting of the small muscles 
of the hand. These mild glomerular changes are described as affecting only a small number of glomeruli. ${ }^{(4)}$ However, in the Hammersmith series of 13 renal biopsy specimens, 11 showed focal glomerulonephritis, with necrotising features present in eight and crescents in nine. ${ }^{(7)}$ By IF, areas of necrosis in the glomeruli may contain IgM, C3 and fibrinogen. ${ }^{(2,7,8)}$ One report revealed immunoglobulin A staining in the glomerulus. ${ }^{(3,6)}$ Both peripheral blood and tissue eosinophilia are major manifestations of the syndrome, and the majority of patients tested have increased IgE levels. ${ }^{(9)}$ The ACR criteria we used to make a diagnosis of CSS are the presence of: (a) asthma; (b) eosinophilia $>10 \%$; (c) mononeuritis multiplex; (d) transient pulmonary infiltrates; (e) paranasal sinus abnormality; and (f) extravascular eosinophilic tissue infiltration. The fulfilment of any four of the above six criteria confirms the diagnosis of CSS, as in the present case.

Notably, presentation of renal involvement in CSS is more subtle. ${ }^{(10)}$ Reviewing the results at Hammersmith Hospital, Clutterbuck et al reported that renal involvement, detected in 16 out of 19 patients with CSS, was frequently severe. ${ }^{(7)}$ Microscopic haematuria was present in 13 patients, granular casts in nine, and red cell casts in three. 12 patients had proteinuria, and three out of these 12 patients had nephrotic syndrome. Four patients presented with a serum creatinine level of $424 \mu \mathrm{mol} / \mathrm{L}$. Among these four patients, two were dialysis-dependent at the time of presentation. The common notion regarding renal vasculitis from the onset is that the initial clinical presentation would be rapidly progressive glomerulonephritis, which was not the case in our patient. ${ }^{(10)}$

It is unfortunate that CSS in the early stages is often underdiagnosed. If detected early, CSS would be more responsive to corticosteroid therapy, while cases with advanced vasculitis may require the addition of more powerful immunosuppressive drugs. ${ }^{(11)}$ Our patient was put on intravenous methylprednisolone, followed by oral steroids. He was initially prescribed oral cyclophosphamide, as per conventional textbook guidelines of the management of CSS. However, the patient did not receive any clinical or biochemical benefit, and we decided to switch his treatment to intravenous cyclophosphamide. This showed excellent results with considerable disability limitation and a significant drop in eosinophil count. He continued taking oral steroids and cyclophosphamide, and his fever and diarrhoea had subsided. He was put on a cock-up splint for his wrist drop and was doing well at the time of this writing.

As CSS is not curable and relapses or flares are common, it is very important that the disease is carefully monitored by a physician throughout its course, with regularly scheduled laboratory tests even when in remission. ${ }^{(3)}$ CSS is a chronic and sometimes life-threatening disease, but with effective medical care, most patients can have a good quality of life. In a study by Sinico et al, ${ }^{(12)} 11$ out of 16 cases of CSS that underwent kidney biopsy showed features of necrotising crescentic glomerulonephritis, of which all were $p$-ANCA positive.

In summary, we have reported a rare case of CSS with an uncommon renal involvement, which was treated unconventionally. In this case, renal involvement was not in the form of rapidly progressive glomerulonephritis but focal segmental glomerulosclerosis, which is an uncommon clinical correlation in this disease scenario. Apart from that, the patient's renal vasculitis did not manifest with nephritic or nephritic range proteinuria, which is again a rarity, and his nephritis responded to an unconventional mode of therapy.

\section{REFERENCES}

1. Harrold LR, Andrade SE, Go AS, et al. Incidence of Churg-Strauss syndrome in asthma drug users: a population-based perspective. J Rheumatol 2005; 32:1076-80.

2. Barbiano di Belgiojoso G, Genderini A, Sinico RA, et al. Acute renal failure due to microscopic polyarteritis with the same histological and clinical patterns in a father and his son. Contrib Nephrol 1991; 94:107-14.

3. Schmitt WH, Csernok E, Kobayashi S, et al. Churg-Strauss syndrome: serum markers of lymphocyte activation and endothelial damage. Arthritis Rheum 1998; 41:445-52.

4. Solans R, Bosch JA, Pérez-Bocanegra C, et al Churg-Strauss syndrome: outcome and long-term follow-up of 32 patients. Rheumatology (Oxford) 2001; 40:763-71.

5. Nochy D, Daugas E, Droz D, et al. The intrarenal vascular lesions associated with primary antiphospholipid syndrome. J Am Soc Nephrol 1999; 10:507-18.

6. Churg J, Strauss L. Allergic granulomatosis, allergic angiitis, and periarteritis nodosa. Am J Pathol 1951; 27:277-301.

7. Clutterbuck EJ, Evans DJ, Pusey CD. Renal involvement in Churg-Strauss syndrome. Nephrol Dial Transplant 1990; 5:161-7.

8. Wechsler ME, Garpestad E, Flier SR, et al. Pulmonary infiltrates, eosinophilia, and cardiomyopathy following corticosteroid withdrawal in patients with asthma receiving zafirlukast. JAMA 1998; 279:455-7.

9. Lanham JG, Elkon KB, Pusey CD, Hughes GR. Systemic vasculitis with asthma and eosinophilia: a clinical approach to the Churg-Strauss syndrome. Medicine (Baltimore) 1984; 63:65-81.

10. Tesar V, Ríhová Z, Jancová E, Rysavá R, Merta M; European randomized trials. Current treatment strategies in ANCA-positive renal vasculitislessons from European randomized trials. Nephrol Dial Transplant 2003; 18 (Suppl 5):v2-4.

11. Cooper BJ, Bacal E, Patterson R. Allergic angiitis and granulomatosis. Prolonged remission induced by combined prednisone-azathioprine therapy. Arch Intern Med 1978; 138:367-71.

12. Sinico RA, Di Toma L, Maggiore $U$, et al. Renal involvement in Churg-Strauss syndrome. Am J Kidney Dis 2006; 47:770-9. 